\title{
Second-order Bandpass Frequency Selective Surface for Terahertz Applications
}

\author{
Amir Ebrahimi, Student Member, IEEE, Withawat Withayachumnankul, \\ Said Al-Sarawi, Member, IEEE, and Derek Abbott, Fellow, IEEE \\ School of Electrical and Electronic Engineering, The University of Adelaide, SA 5005, Australia \\ amir.ebrahimi@adelaide.edu.au
}

\begin{abstract}
In this paper, a two-layered frequency selective surface with miniaturized elements is proposed for millimeter wave and terahertz applications. The advantages of this design are a harmonic free transmission response and low sensitivity of its frequency response to the angle of incidence. Performance of the designed structure is verified using full-wave electromagnetic simulations. The results show a second-order filter response with a center frequency of $0.4 \mathrm{THz}$ with the fractional bandwidth of $25 \%$ and more than $25 \mathrm{~dB}$ out of band rejection.
\end{abstract}

\section{INTRODUCTION}

$\mathbf{N}$ OWADAYS, research on devices for high performance millimeter wave and terahertz applications attracts significant interest due to a rapid growth in the use of terahertz radiation in imaging, spectrometry, molecular sensing, remote sensing, etc. Bandpass frequency selective surfaces (FSSs) are useful components in these applications, yet the availability and performance are limited. A common feature among the conventional FSS designs is that the dimensions of unit cell resonant elements are comparable to the operation wavelength. This feature makes their frequency response sensitive to the angle of incidence [1]-[3]. Therefore, in some applications FSSs with subwavelength unit cells are favorable due to their low-sensitivity to the incidence angle [4], [5]. In this paper, a double layered FSS is designed with the unit-cell dimensions much smaller than the operation wavelength. This miniaturized structure decreases the sensitivity of the FSS to oblique angles of incidence. Furthermore, this miniaturized structure is capable of suppressing unwanted higher order harmonics.

\section{FSS TOPOLOGY}

Fig. 1 shows the unit cell of the designed THz FSS. The front layer of the unit cell is composed of a capacitive metallic patch enclosed by a metallic loop. The back layer of the unit cell is made of a Jerusalem cross resonator surrounded by a metallic loop. These metallic arrays are considered to be patterned on the two sides of a $135 \mu \mathrm{m}$ thick quartz substrate. The front and back metallization is $0.2 \mu \mathrm{m}$ thick gold, well above the skin depth at terahertz frequencies.

The proposed FSS can be modelled by an equivalent circuit model shown in Fig. 2. In this model, the front layer of the FSS is modelled with a parallel $L_{1} C_{1}$ resonator where, $L_{1}$ models the inductive behavior of the front layer wire grid and $C_{1}$ models the capacitive effect between the wire grid and the square patches. The back layer is modelled

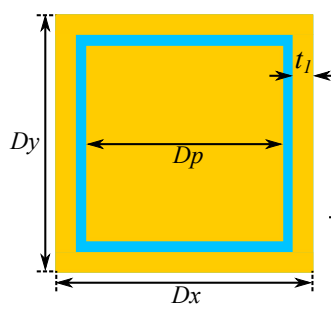

(a)

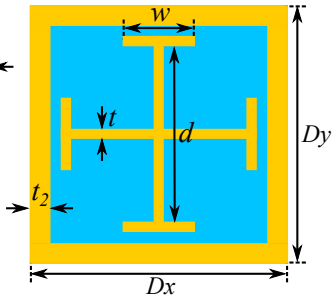

(b)
Fig. 1. Unit cell of the designed THz FSS. (a) Front layer. (b) Back layer. Dimmenssions are: $D_{x}=D_{y}=175 \mu \mathrm{m}, D_{P}=120 \mu \mathrm{m}, t_{1}=22.5 \mu \mathrm{m}$, $t_{2}=22.5 \mu \mathrm{m}, d=110 \mu \mathrm{m}, t=5 \mu \mathrm{m}, w=25 \mu \mathrm{m}$.

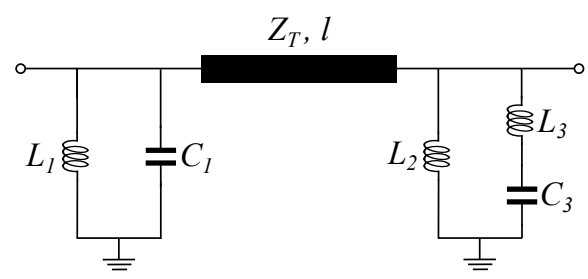

Fig. 2. Equivalent circuit model of the proposed FSS. The element values are: $L_{1}=9.3 \mathrm{pH}, C_{1}=25 \mathrm{fF}, L_{2}=70 \mathrm{pH}, L_{3}=25 \mathrm{pH}, C_{2}=2.5 \mathrm{fF}$, $Z_{T}=169 \Omega$ and $l=150 \mu \mathrm{m}$.

as a hybrid resonator consisting of $L_{2}$ inductor in parallel with $L_{3} C_{3}$ series resonator. The $L_{2}$ inductor represents the wire grid of the back layer and the $L_{3} C_{3}$ series resonator describes the Jerusalem cross resonator. In addition, the quartz substrate that separates the two metallic layers is modelled as a section of a transmission line with the length of $135 \mu \mathrm{m}$ and a characteristic impedance of $Z_{T}=Z_{0} / \sqrt{\epsilon_{r}}$, where $Z_{0}=377 \Omega$ is the free space impedance and $\epsilon_{r}$ is the dielectric constant of the quartz substrate. The equivalent circuit parameters obtained from the closed-form equations and the curve fitting of EM and circuit simulations are listed in the caption on Fig. 2.

\section{RESUltS}

The designed FSS and its equivalent circuit model are simulated by using the parameters given in Fig. 1 and Fig. 2. Fig. 3 shows the transmission response $\left(S_{21}\right)$ of the FSS obtained from the full-wave electromagnetic simulation together with its circuit model simulation. As seen, there is a good agreement between the EM and circuit model simulations confirming the validity of the circuit model. The bandpass characteristic is 


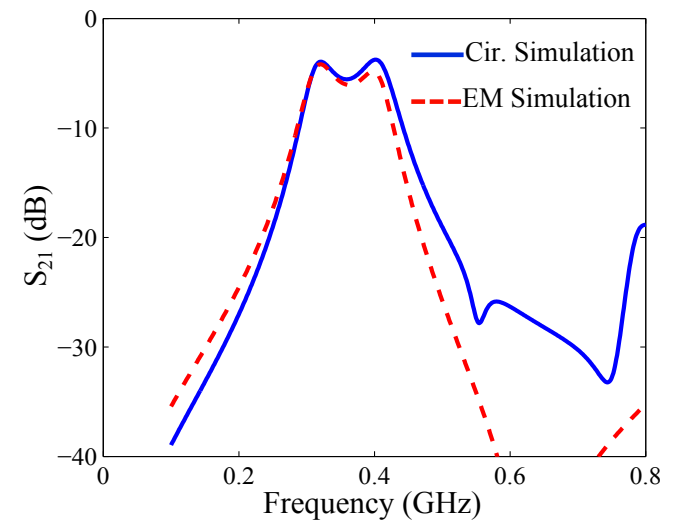

Fig. 3. Comparison between the full-wave electromagnetic and circuit model simulation of the proposed FSS.

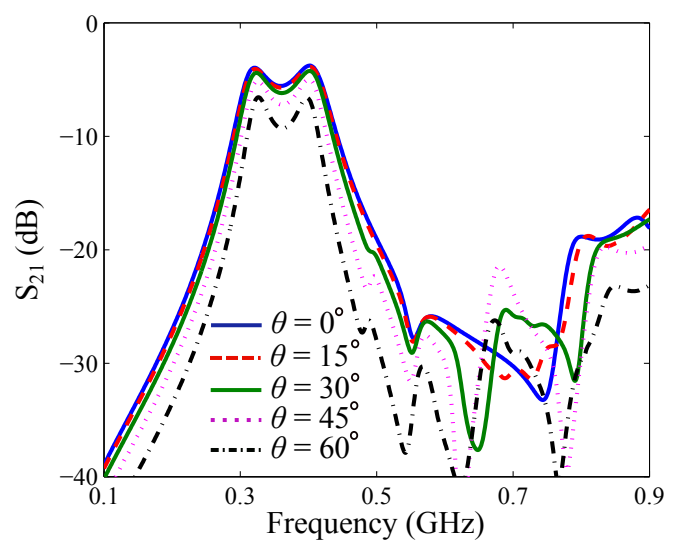

Fig. 4. Transmission response of the designed FSS for TE polarization of the incident wave at different incidence angles $\left(\theta=0^{\circ}\right.$ represents the normal incidence).

achieved with a center frequency around $0.4 \mathrm{THz}$. As seen, the filter has no harmonic response up to $0.8 \mathrm{THz}$. There is a zero in the transmission response of the FSS at around $0.6 \mathrm{THz}$, which improves the out of band rejection of the filter. From the circuit model in Fig. 2, this transmission zero takes place at the resonance frequency of the back layer series resonator, which can be described as

$$
f_{\mathrm{z}}=\frac{1}{2 \pi \sqrt{L_{3} C_{3}}} .
$$

Based on (1), the transmission zero frequency can be tuned by changing the value of $L_{3}$ and $C_{3}$. From the back layer unit cell in Fig. 1(b), the value of $L_{3}$ can be tuned by changing $d$ and $t$. Increasing $d$ or decreasing $t$ result in a larger $L_{3}$ and vice versa. Additionally, increasing $w$ or $d$ will increase the value of $C_{3}$. The slope of the upper and lower sides of the passband is around $70 \mathrm{~dB} /$ octave, showing a good selectivity of the proposed filter.

One important feature in designing of FSSs is the immunity of the frequency response respect to the oblique incidence angles. Fig. 4 shows the transmission response of the designed FSS for the transverse electric (TE) polarization of the incident wave at different incidence angles. The frequency response

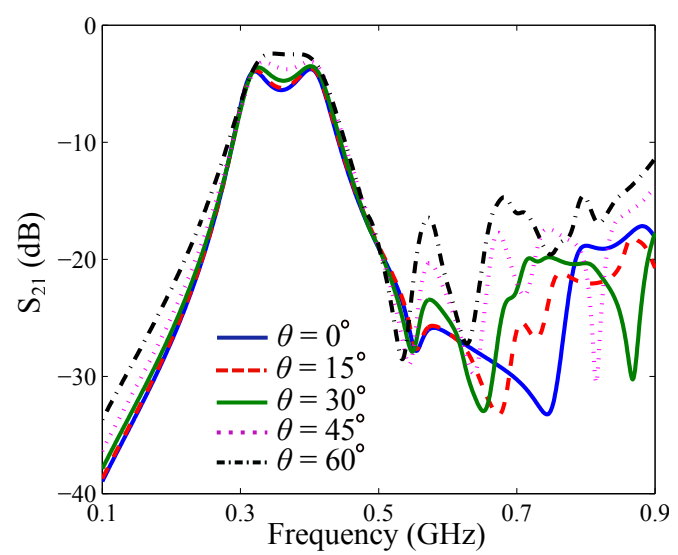

Fig. 5. Transmission response of the designed FSS for TM polarization of the incident wave at different incidence angles $\left(\theta=0^{\circ}\right.$ represents the normal incidence).

of the FSS for the transverse magnetic (TM) polarization is demonstrated in Fig. 5 at different incidence angles. The structure shows the same frequency response for both of the TE and TM polarizations at normal incidence angle due to the fourfold symmetric structures of the front and back layers. However, by increasing the incidence angle, the responses for the TE and TM polarizations will change. This feature is mainly caused by the change of wave impedances for the TE and $\mathrm{TM}$ polarizations $\left(Z_{0, \mathrm{TE}}=Z_{0} / \cos \theta, Z_{0, \mathrm{TM}}=Z_{0} \cos \theta\right)$. So, the wave impedance for the TE polarization increases with increasing the incidence angle resulting in a smaller bandwidth for the TE response. On the other hand, the wave impedance of the TM polarization will be decreased by increasing the incidence angle, which results in a larger bandwidth for the TM transmission response [6].

\section{SUMMARY}

A second-order bandpass terahertz FSS has been designed and simulated. An equivalent circuit model is developed for better insight into the FSS frequency response. The filter shows a good selectivity in the passband. Further, the response is harmonic-free up to $0.8 \mathrm{THz}$. The FSS shows a good immunity with respect to oblique angles of the incident wave.

\section{REFERENCES}

[1] M. Moallem and K. Sarabandi, "Miniaturized-element frequency selective surfaces for millimeter-wave to terahertz applications," IEEE Transactions on Terahertz Science and Technology, vol. 2, no. 3, pp. 333-339, 2012.

[2] M. Li and N. Behdad, "A third-order bandpass frequency selective surface with a tunable transmission null," IEEE Transactions on Antennas and Propagation, vol. 60, no. 4, pp. 2109-2113, 2012.

[3] A. Rashid, Z. Shen, and B. Li, "An elliptical bandpass frequency selective structure based on microstrip lines," IEEE Transactions on Antennas and Propagation, vol. 60, no. 10, pp. 4661-4669, 2012.

[4] A. Ebrahimi, W. Withayachumnankul, S. Al-Sarawi, and D. Abbott, "Design of dual-band frequency selective surface with miniaturized elements," in IEEE International Workshop on Antenna Technology (iWAT), 2014, pp. 206-209.

[5] A. Ebrahimi, P. Yaghmaee, W. Withayachumnankul, C. Fumeaux, S. AlSarawi, and D. Abbott, "Interlayer tuning of X-band frequency-selective surface using liquid crystal," in Microwave Conference Proceedings (APMC), 2013 Asia-Pacific, 2013, pp. 1118-1120.

[6] N. Behdad, "A second-order band-pass frequency selective surface using nonresonant subwavelength periodic structures," Microwave and Optical Technology Letters, vol. 50, no. 6, pp. 1639-1643, 2008. 